Erschienen in: Harras, Gisela (Hrsg.): Die Ordnung der Wörter. Kognitive und lexikalische Strukturen. - Berlin, New York: de Gruyter, 1995. S. 289-302. (Institut für deutsche Sprache. Jahrbuch 1993)

REGINA HESSKY

\title{
Zum kognitiven Ansatz in der Phraseologie: „Aufgewärmter Kohl” oder „eine neue Platte"?
}

\section{Themenwahl, Zielsetzung und Abgrenzung}

1.1 Die unmittelbare Anregung, über dieses Thema zu sprechen, kam von den Veranstaltern der Tagung, und ich betrachtete es als eine Art Herausforderung an die Phraseologie, in dem durch das Rahmenthema gegebenen Kontext eine Bestandsaufnahme zu machen, und zwar in zweifacher Beziehung. Einerseits mit dem Ziel zu klären, in welchen Bereichen der Phraseologieforschung und unter welchen Aspekten bereits erfolgversprechende Versuche einer kognitiv orientierten Betrachtung dieser in vielerlei Hinsicht spezifischen sprachlichen Erscheinung unternommen worden sind. Andererseits, um zu zeigen, welche Fragestellungen m.E. besonders geeignet sind, in diesem Rahmen untersucht zu werden, weil man dadurch mit einem Erkenntniszuwachs rechnen kann.

Ich beschränke mich allerdings darauf, von bestimmten Fragestellungen der Phraseologieforschung ausgehend zu bestimmten Thesen/Hypothesen/Erkenntnissen der Kognitiven Linguistik eine Beziehung herzustellen. So hoffe ich zeigen zu können, daß ein kognitiver Ansatz in der Phraseologie nicht nur legitim, sondern durchaus nutzbringend sein kann.

Mit dem vielleicht nicht sehr gelungenen Untertitel vom aufgewärmten Kohl und der neuen Platte sollte lediglich angedeutet werden, daß diese Problematik seit gar nicht so kurzer Zeit in der Luft liegt, und daß in den letzten Jahren mehrere Arbeiten veröffentlicht worden sind, die es verdienen - wenngleich in verschiedenen Dimensionen - unter kognitivem Aspekt beachtet zu werden. Insofern werde ich mich durchaus auch „mit fremden Federn schmücken", indem ich bestimmte einschlägige Arbeiten referiere. Darüber hinaus erwecken manche Erkenntnisse der Kognitiven Linguistik auch bestimmte Reminiszenzen an Erkenntnisse aus früherer Zeit, die seinerzeit wenig beachtet oder später verdrängt und vergessen worden waren. Auf einige will ich an entsprechender Stelle verweisen.

1.2 Ich halte es für notwendig, im Vorfeld auch zu klären, was dieser Beitrag - aus verschiedenen Gründen - nicht leisten kann: Erstens maße ich mir nicht an, zu offenen, kontroversen Fragen und verschiedenen Ausprägungen der Kognitiven Linguistik Stellung zu nehmen, und zweitens ist es auch nicht mein Ehrgeiz, eine "kognitive Theorie" der Phraseologie zu entwickeln. Daher dürfte eine bestimmte Inkonsistenz und Inkohärenz, wenn auch nicht angemessen, so doch tolerierbar sein, 
die sich teilweise auch im Eklektizismus der Terminologie und in der Heterogenität des Begriffsapparates bemerkbar machen wird.

1.3 Schlieblich seien einige Grundbegriffe geklärt, die ich verwenden werde:

- Mit Phraseologie, Phraseologismus, Phraseolexem ist im Zusammenhang dieses Beitrags und Themas der Kernbereich der Phraseologie, d.h. die traditionell als Idiomatik/Idiome bezeichnete Subklasse angesprochen, für die eine (von der wörtlich-literalen verschiedene, mit dieser jedoch metaphorisch zusammenhängende) figurative, d.h. phraseologische Bedeutung charakteristisch ist, z.B.: ain Ruder sein, mit offenen Karten spielen, auf keinen grünen Zweig kommen usw.

- Unter konzeptuellen Strukturen verstehe ich - in Anlehnung an Monika Schwarz - elementare Einheiten der strukturellen Kognition, mentale Organisationseinheiten, die die Funktion haben, Wissen über die Welt zu speichern. Die Konzepte sind im Gedächtnis durch verschiedene Relationen mit anderen Konzepten verknüpft, und durch die interkonzeptuellen Beziehungen entstehen kognitive Strukturen als mentale Repräsentationen der äuBeren Welt (Schwarz 1992, S. 84ff.).

- Im Einklang mit Lakoff/Johnson gehe ich davon aus, daß die konzeptuellen Strukturen weitgehend metaphorisch strukturiert sind und uns infolge ihrer Systematik ermöglichen, Ausdrücke, d.h. die Versprachlichungen eines Konzepts zu verwenden, um aufgrund von Ähnlichkeitsrelationen über ein anderes Konzept zu sprechen (Lakoff/Johnson 1980, S. 52).

\section{Ausgangsposition}

Die Erwartung, das Erklärungspotential der Kognitiven Linguistik würde auch im Hinblick auf die phraseologischen Ausdrucksmittel natürlicher Sprachen zu einem Erkenntniszuwachs führen, liegt begründet in ihrer Selbstbestimmung nicht als spezielles Teilgebiet der Linguistik, sondern - in Bierwischs Formulierung - als ,... ein bestimmtes Verständnis vom Charakter und der Zielstellung der Wissenschaft von der natürlichen Sprache" (Bierwisch 1987, S. 645) zum einen sowie in dem Übergang von einer beschreibenden zu einer erklärenden Wissenschaft als fundamentaler Wechsel ihrer Orientierung (Bierwisch 1987, S. 646) zum anderen.

Aus der zentralen Annahme des kognitiven Ansatzes, der kognitiven Betrachtung der Sprache, „daB alle sprachlichen Phänomene [...] Sprachreflexe der entsprechenden kognitiven Strukturen sind" (Bara- 
nov/Dobrovol'skij 1991, S. 112), läßt sich die Folgerung ableiten, daß auch „allen Erscheinungen der semantischen Derivation reguläre Transformationsregeln zugrundeliegen, und zwar eines Prototyps (im Sinne der Gestalttheorie)" (Baranov/Dobrovol'skij 1991, S. 116). M.a.W.: Da Phraseolexeme durch semantische Derivation (d.h. sekundäre Nomination) entstehen, muß der Zusammenhang zwischen kognitiver Struktur und Sprachreflex auch für diese Ausdrucksmittel gültig sein, und damit müssen auch Phraseolexemen reguläre Transformationsregeln zugrundeliegen.

Aus dem Postulat eines solchen Zusammenhangs folgt, daß die adäquate Untersuchung der Phraseologie einen Zugang zu diesen kognitiven Strukturen ermöglicht (Lakoff/Johnson 1980, S. 7). Wie es Durčo sieht:

\footnotetext{
„Für eine kognitiv orientierte Linguistik, die sich bewußt eben mit dem Zusammenhang $z$ wischen der modellhaften Vorstellung, realem Gebrauch und Verstehen von sprachlichen Einheiten befaßt, stellen die idiomatischen Erscheinungen als sekundäre Gebilde der Sprache ein besonders wertvolles, aber bis heute nicht entsprechend bewertetes $\mathrm{Ma}$ terial dar. [...] ohne Berücksichtigung der idiomatischen Erscheinungen kanu man kaum ein angemessenes Bild einer natürlichen Sprache erzielen."

(Durčo 1990, S. 2)
}

2.1 Ein kurzer Blick auf die Forschungsgeschichte zeigt, daß es in der Phraseologie bereits bestimmte Entwicklungstendenzen und Forschungsansätze gibt, die es verdienen, unter kognitivem Aspekt beachtet zu werden. Von diesen werde ich die Problematik der phraseologischen Bedeutung, die der lexikographischen Kodifizierung nach dem ideographischen Prinzip sowie die Modifikation kurz umreißen und schließlich den in der kontrastiven Phraseologie wichtigen Begriff der Ähnlichkeit problematisieren. (Eine ausführliche Erörterung ist in diesem Rahmen natürlich nicht möglich, ich hoffe aber, daß die Perspektiven eines kognitiven Ansatzes aufgezeigt werden können.)

2.2 Auf die Rolle von allgemeinen Denkschemata, Analogisierungen und Metaphorisierungsprozessen sowie diesen zugrundeliegenden gedanklichen Operationen für die Entstehung und das Verstehen von Phraseolexemen wurde bereits vor einigen Jahren von Gréciano hingewiesen: „In Anbetracht der sehr aktuellen Erkenntnisse über mentale Phänomene [...] scheint es verheißungsvoll, das semantische Verhalten des lebendigen Idioms unter diesem Blickwinkel zu erklären." (Gréciano 1988, S. 36)

Auch der "Modellierungsgedanke" ist in der phraseologischen Literatur seit längerem bekannt, und es wurden verschiedene Versionen der Modellierung vorgeschlagen (Černyševa 1980, Raichštejn 1980, Fleischer 1982). 
Wie Baranov/Dobrovol'skij schreiben: „Die Versuche, bestimmte Regularitäten bei der Phrasembildung bzw. Phraseologisierung aufzudecken und auf entsprechende kognitive und linguistische Gesetzmäßigkeiten zurückzuführen, sind vor allem mit dem Begriff der Modellierbarkeit von Phraseologismen verbunden" (Baranov/Dobrovol'skij 1991, S. 113). Im Mittelpunkt steht die Erkenntnis, daß zwischen der wörtlich-literalen und der figurativen, d.h. phraseologischen Bedeutung eine Beziehung besteht (traditionell Motiviertheit genannt), die trotz Unvorhersagbarkeit nicht völlig willkürlich ist, sondern bestimmten Gesetzmäßigkeiten des logisch-assoziativen Denkens folgt.

Insofern ist der Gedanke, semantische Prozesse in der Phraseologie zu modellieren, nicht völlig neu. Neu ist hingegen der Versuch, die bisherigen Grenzen der Modellierung $z u$ überwinden und in die mentale (kognitiv-konzeptuelle) Sphäre vorzudringen bzw. diese in die Modellierung mit einzubeziehen. Einen solchen Versuch, die Phraseologie genauer die Idiome - auf kognitiver Basis zu modellieren, haben Baranov/Dobrovol'skij (1991) vorgelegt.

Die Modellierung wird ermöglicht durch das Postulat der regulären Transformationsregeln, was allerdings im Zusammenhang der Phraseologie nicht Regularität der Generierung von Phraseolexemen meint, also nicht als Möglichkeit und Grundlage der Entwicklung produktiver Modelle ihrer Bildung verstanden werden kann: „Regularität der Transformationsregeln" bedeutet (lediglich) die Möglichkeit und Grundlage der modellhaften Beschreibung bereits lexikalisierter Phraseolexeme im Rahmen eines in der Kognitiven Linguistik entwickelten Konzeptes.

Der besondere Reiz eines solchen Herangehens liegt in der Natur des phraseologischen Sprachzeichens begründet. Wie gesagt, Phraseolexeme sind als Produkte der sekundären Nomination zu einem großen Teil ebenfalls metaphorischen Ursprungs bzw. in der Gegenwartssprache als usualisierte (Mehrwort-)Metaphern begreifbar. „Die Phraseologismen unterscheiden sich von den übrigen sprachlichen Phänomenen u.a. dadurch, daß ihre innere Form gestattet, den Charakter der Transformationen der Zusammenfaltung und Aktualisierung konzeptueller Strukturen in den Sprechakten zu analysieren" (Baranov/Dobrovol'skij 1991, S. 122). Dadurch eignen sie sich für Analysen, die uns zeigen können, wie konzeptuelle Strukturen im Zuge der Versprachlichung zerlegt werden, und wie die Versprachlichung zweier konzeptueller Strukturen auf der Basis einer Kongruenz (als Ähnlichkeitsrelation) erfolgen kann:

(1) ein Schlag unter die Gürtellinie

(2) das Handtuch werfen 
Die Beispiele kann man ansehen als die Versprachlichung zweier „Knoten” des Frames (Skripts) „Boxwettkampf/Boxen als Sportwettkampf". Beide sind analoge (bildhafte) Repräsentationen. (1) erfaBt die Verletzung einer wichtigen Regel, einen nicht fairen, nicht zugelassenen Schlag. (2) fixiert den möglichen Endknoten, das Aufgeben, vorzeitige Beenden aus welchem Grund auch immer. Wenn nun der Sprecher (1) und (2) aus dem (konkreten) Quellenbereich herausgreift und (1) im Sinne von 'unfaires, unerlaubtes Handeln', (2) im Sinne von 'aufgeben' auf einen beliebigen Wettkampf anderer Art, etwa eine Diskussion/Debatte oder einen Konkurrenzkampf 2.B. in der Wirtschaft überträgt, so ist dies das Ergebnis einer bestimmten mentalen Operation: Der Sprecher hat eine Ähnlichkeitsrelation zwischen dem Skript "Boxwettkampf/Boxen als Sportwettkampf" einerseits und dem Skript "Diskussion/Debatte” bzw. „Konkurrenzkampf” andererseits erkannt und das Konkretere auf das Abstraktere bezogen, zur Versprachlichung des Abstrakteren Versprachlichungen des Konkreten herangezogen.

2.3 In diesem sehr vagen und nur in ganz groben Zügen skizzierten Rahmen ließe sich auch eine andere "phraseologiespezifische” Erscheinung besser in den Griff bekommen, die sog. Modifikation als Remotivation, d.h. „die zusätzliche fakultative Wiederbelebung wörtlicher Reminiszenzen, die sprecher- und situationsbedingte Transparentmachung demotivierter Phraseme bzw. Phrasemformative als Reaktualisierung von Referenz" (Gréciano 1991, S. 92). Es geht hier um die vom regulären/normgerechten Gebrauch abweichende, sehr stark kontext- und situationsgebundene Idiomverwendung, die in bestimmten Textsorten besonders häufig auftritt (Presse, insbesondere Schlagzeilen, Werbesprache, Sprachwitze).

Die These von den konzeptuellen Strukturen metaphorischen Charakters, von der metaphorischen Verbindung konkreter mit abstrakten Strukturen scheint auch für diese Erscheinung einen geeigneten Erklärungsrahmen darzustellen, sowohl unter dem Aspekt der Rezeption wie auch der Produktion solcher Modifikationen.

Bei den verschiedenen Ausprägungen dieser Erscheinung, deren erste detaillierte Beschreibung sich bei Burger/Buhofer/Sialm (1982) und die bislang wohl umfassendste bei B. Wotjak (1992) findet, geht es (in der Regel) darum, daB der Sprecher okkasionell, kontext- und kotextbedingt zurückgreift auf die konzeptuelle Struktur (den Quellenbereich), aus welcher das Phraseolexem metaphorisch „entlehnt” wurde, und daß bestimmte andere Elemente - mögliche „Knoten” - dieses konkreten Konzepts auf das abstrakte Konzept (den Zielbereich) übertragen werden. Die Quelle ist eine mentale Operation komplexer oder weniger komplexer 
Art: Die - gegebenenfalls bereits verdunkelte - Identitäts-/Ähnlichkeitsbeziehung zwischen den Konzepten A und B (dem Quellen- und Zielbereich) wird bewußt und okkasionell erweitert auf einen oder mehrere „Knoten” der beiden Frames. Man könnte hier von einer neuen, durchaus okkasionellen, Metaphorisierung sprechen, die in umgekehrter Richtung verläuft, indem sie vom Abstrakten ausgeht - oder anders formuliert: In der Modifikation wird das Konzept A (der Quellenbereich) neu entdeckt (Remotivation!), und aufgrund einer neuen Kongruenz/Ähnlichkeitsbeziehung wird eine neue - okkasionelle - Versprachlichung dieser metaphorischen Beziehung vorgenommen.

(3) „Der offene Fragenkatalog, der u.a. Probleme des Spracherwerbs und der Didaktisierung sowie auch konversationsanalytische Ansätze ethnomethodologischer Prägung ausspart, macht deutlich, daß die Phraseologie - nicht nur aus sprachvergleichender Sicht - noch immer ein weites Feld ist, das es zu erkunden und zu beackern sich lohnt."

(B. Wotjak 1992, S. 212)

Im Hintergrund dieser Modifikation wird ein weites Feld als 'schwer überschaubarer Bereich' durch die Kollokation Feld beackern zurückgeführt auf die konzeptuelle Struktur „den Boden bestellen”. Der ko(n)textuelle Rahmen blockiert jedoch eine Rezeption als Versprachlichung dieses Konzeptes, und so muß der Rezipient durch Rückgriff auf entsprechende mentale Operationen den "Knoten" „beackern” wiederum ins abstrakte Frame - etwa „Bewältigen” - überführen.

(4) "Frauen wurden privat und beruflich zu kompetenten Ansprechpartnern und Gesprächspartnern. Sie stehen ihre "Frau" und nehmen die ihnen angetragenen oder von ihnen ausgewählten Aufgaben genauso qualifiziert wahr wie ihre männlichen Kollegen."

(Süddeutsche Zeitung, 25/26.11.1989)

Beispiel (4) repräsentiert einen anderen Typ der Modifikation: eine Konstituente des Idioms seinen Mann siehen ist substituiert, ohne daß dadurch die phraseologische Bedeutung 'auf sich gestellt tüchtig sein und sich bewähren' aufgehoben würde. Durch diese Substitution (Mann durch Frau) wird das wohl bereits verdunkelte Bild mit dem Mann als "Krone der Schöpfung” zeitweilig bewußt gemacht, zugleich aber auch korrigiert bzw. erweitert. Dieses Beispiel, daß sich von (3) im Fehlen einer sinnvollen literalen Bedeutung unterscheidet, scheint mir - ausgehend vom Kontext, in dem es um die gesellschaftliche Stellung und Rolle der Frau geht - durchaus nachvollziehbar zu sein. Das primäre, ursprüngliche, historisch-sozial geprägte (und engere) "Mann-Konzept" wird erweitert und mit dem „Frau-Konzept” verbunden. 
Die zentrale Rolle der Metapher und des metaphorischen Denkens als Triebkraft der Entwicklung der Sprache ist kein völlig neuer Gedanke. So sprach z.B. bereits Herder vom "Metapherngeist" der Sprache: „Man nahm Begriffe, die nicht sinnlich waren, in die Sprache; man nannte sie aber, wie von selbst $\mathrm{zu}$ vermuten ist, mit bekannten sinnlichen Namen. Daher müssen die ersten Sprachen bildervoll und reich an Metaphern gewesen sein." (Herder 1952, S. 384)

Bei Walter Porzig liest man: „Die Sprache übersetzt alle unanschaulichen Verhältnisse ins Räumliche. [...] Diese Eigentümlichkeit gehört zu den unveränderlichen Zügen (<Invarianten $>$ ) der menschlichen Sprache." (Porzig 1986, S. 209-210)

Iván Fónagy entwickelte ein Modell sprachlicher Entwicklung und stellte fest: „Es scheint wahrscheinlich zu sein, daß die Metapher ein Modell bietet, das bei jeglicher sprachlicher Veränderung anwendbar ist." (Fónagy 1978, S. 89)

2.4 In der langen Geschichte der Lexikographie hat es mehrfach Versuche gegeben, das Lexikon (einschließlich der Phraseologie) nicht in alphabetischer Anordnung nach dem semasiologischen, sondern nach dem onomasiologischen Prinzip zu präsentieren. Die meisten kritischen Einwände gegen solche Nachschlagewerke gipfeln im Hinweis darauf, daß sich eine angemessene Strukturierung der außersprachlichen Wirklichkeit, die als Grundlage für die Anordnung des Sprachmaterials dienen könnte, schwer finden läßt. Speziell zu Ursachen für die Unzulänglichkeiten der onomasiologischen Erfassung des phraseologischen Materials haben sich in jüngster Zeit u.a. Dobrovol'skij (1992a), Möhring (1992) geäußert.

Es hat sich gezeigt, daß man bei der Kodifizierung der Phraseologie noch weniger als im übrigen Bereich des Lexikons, von einem apriori entwickelten - deduktiven - Begriffssystem ausgehen kann, da die Bereiche, Sachgebiete, Begriffsfelder, für die es phraseologische Ausdrücke gibt, von Sprache zu Sprache Unterschiede aufweisen. Selbst im Falle einer Versprachlichung können in der "Dichte”, Verteilung, Differenzierung erhebliche Divergenzen bestehen: Es gehört zu den trivialen Feststellungen, daß es Phraseolexeme weniger für den nominalen als für den „verbalen” Bereich, also als semantische Prädikate, vor allem für Einstellungen, Wertungen etc. gibt, und kraft des euphemistischen Charakters zahlreicher Phraseolexeme die negativen Einstellungen, Wertungen überwiegen.

Man müßte also offensichtlich den umgekehrten Weg gehen, d.h. die aus der Bedeutung der Phraseolexeme gewonnenen „begrifflichen Kerne” inventarisieren, von diesen ausgehend ein „Bezugssystem” entwickeln und 
dieses als Anordnungsprinzip verwenden. Ein Katalog der entsprechenden konzeptuellen Strukturen mit ihrem Prototyp könnte die Anordnung der Phraseolexeme ermöglichen, so, wie der Sprecher sie in seinem mentalen Lexikon speichert.

Mängel und Unzulänglichkeiten der alphabetischen Anordnung unter dem Aspekt der Erfassung des (gesamten) phraseologischen Sprachmaterials treten auch in der zweisprachigen Phraseographie deutlich zutage: Im Falle der semasiologischen Anordnung können bei einem beliebigen Sprachenpaar in beiden Richtungen lediglich nur die Phraseolexeme der jeweiligen Ausgangssprache erfaßt und diesen ihre Äquivalente in der jeweiligen Zielsprache zugeordnet werden. Bekanntlich gibt es aber in jeder Sprache Phraseolexeme, die in einer anderen keine lexikalisierten Äquivalente haben, d.h. aus einem alphabetisch angeordneten zweisprachigen phraseologischen Wörterbuch würde man jeweils nur in der Ausgangssprache das gesamte Material fixieren können.

Aus diesem asymmetrischen Verhältnis folgt, daß es für die zweisprachige Phraseographie von besonderer Bedeutung wäre, ein Anordnungsprinzip zu erarbeiten, das uns ermöglicht, in beiden Sprachen den gesamten phraseologischen Bestand zu erfassen. Außer dem praktischen Nutzen könnten von solchen Darstellungen ausgehend wichtige theoretische Fragen beantwortet werden, wie z.B.: Welches sind die besonders "phraseologiefreundlichen" Denotatsbereiche, wo gibt es phraseologische Reihenbildungen mit funktionalstilistischer Differenzierung usw. (Möhring 1992).

Bei den Bemühungen um eine ideographisch angelegte Erfassung der Phraseologie geht man vom naiven Weltbild des Sprechers als „organisierender Idee” aus. Dobrovol'skij schlägt einen Weg im Sinne der Prototypensemantik in der Version von Rosch und Lakoff vor. In seiner einschlägigen Arbeit skizziert er den Vorgang und verschiedene Techniken der Gewinnung von relevanten Deskriptoren der Idiome, die dann die Grundlage der Kodifizierung darstellen könnten. Dabei ergibt sich eine besondere Schwierigkeit daraus, daß man die Mehrheit der Phraseolexeme nicht auf einen Deskriptor zurückführen kann, zumal ihre Bedeutung meistens - aus verschiedenen Perspektiven betrachtet - verschiedene Deskriptoren zuläßt oder suggeriert. Im Beispiel - es stammt ebenfalls von Dobrovol'skij - bei jm. ins Fettnäpfchen treten läßt die Basisproposition „X ist bei $\mathrm{Y}$ (mit $\mathrm{Z}$ ) ins Fettnäpfchen getreten” eine $\mathrm{X}$ Perspektive - 'Kränkung/Beleidigung' -, eine Y-Perspektive - 'Verärgerung' - und eine Z-Perspektive - 'ungeschickte, unpassende Äußerung' zu (Dobrovol'skij 1992b, S. 6). In den meisten Fällen muß man also von Mehrfachzuordnungen ausgehen. 
3. Motto: „Wer vergleicht, sieht mehr und anderes; es fällt ihm mehr auf, und es fällt ihm mehr ein." (Bausch/Gauger 1971, S. XIII)

Kontrastive Arbeiten zur Phraseologie bestätigen, was jeder, der außer seiner Muttersprache auch (eine) andere Sprache(n) kennt, früher oder später selbst feststellen kann: In ihren phraseologischen Ausdrucksmitteln weisen Sprachen, unabhängig von ihrem genetischen Verwandtschaftsgrad, mehr oder weniger große Ähnlichkeiten auf. Wenn es im Deutschen heiBt: den Wald vor lauter Bäumen nicht sehen, sagt man auf englisch ganz ähnlich not to be able to see the wood for the trees; der Franzose sagt les arbres cachent la foret oder dem deutschen im trüben fischen entspricht englisch to fish in troubled waters bzw. russisch lovit" rybu v mutnoj vode. Die Ähnlichkeit besteht darin, daß dieselbe phraseologische Bedeutung mit in ihrer literalen Bedeutung ähnlichen Syntagmen ausgedrückt wird - m.a.W.: die durch die literale Bedeutung evozierten "Bilder" sind recht ähnlich.

3.1 Man geht in der Regel von drei großen Kategorien der zwischensprachlichen Äquivalenzbeziehung aus: der totalen/vollständigen, der partiellen/teilweisen und der Null-Äquivalenz. Je nach Zielsetzung und Differenziertheit der Vergleichskriterien kann es zur Aufstellung verschiedener Subklassen kommen, und je nach Intention widmet man mal den totalen, mal den Null-Äquivalenten mehr Aufmerksamkeit: Erstere zeigen die Gemeinsamkeiten, letztere unterstützen die Behauptung, daß die Phraseologie "ein Spiegel der nationalen Kultur und Spezifik” ist. Stellvertretend für die zahlreichen kontrastiven Untersuchungen s. u.a. Durčo (1990); Földes (1990), (1991); Gréciano (1988); Korhonen (1991); Raichštejn (1980).

Unabhängig vom Anteil der totalen bzw. partiellen Äquivalenzbeziehungen können wir die phraseologischen Subsysteme der Sprachen als prinzipiell ähnlich bezeichnen. Dieser Ähnlichkeitsbegriff unterscheidet sich allerdings von der Ähnlichkeit im Sinne von partieller Äquivalenz, von der im weiteren die Rede sein wird.

Diese Kategorie, d.h. die Gruppe von Phraseolexemen, die sich als ähnlich bezeichnen lassen im Sinne 'in bestimmten Merkmalen übereinstimmend', hat bislang in der Forschung vergleichsweise geringe Aufmerksamkeit erregt.

Die Ähnlichkeit einzelner Phraseolexeme wird gewöhnlich nach mehreren, verschiedenen Gesichtspunkten ermittelt, und auf dieser Grundlage läßt sich eine Hierarchie der partiellen Äquivalenzbeziehung aufstellen. Diesbezügliche Arbeiten haben sicherlich einen beachtlichen 
theoretischen Erkenntniswert, wiewohl sie auch für bestimmte Anwendungsbereiche - zweisprachige Phraseographie, Fremdsprachendidaktik - von großer Bedeutung sind. Sie gehen aber zumeist kaum über den klassifizierend-systematisierenden Aspekt hinaus, und ich glaube behaupten zu dürfen, daß die kontrastive Phraseologie mit ihren bisherigen Analyseverfahren (und Vergleichskriterien) die Grenze ihrer Möglichkeiten erreicht hat. Eine Erneuerung scheint mir jedoch durch die Hinwendung zur kognitiven Sprachbetrachtung möglich zu sein.

3.2 Als Ursache für die Ähnlichkeit der phraseologischen Ausdrucksmittel weist man in der Regel auf die jahrhundertelangen Kontakte der Sprachgemeinschaften und ihre Zugehörigkeit zum gleichen Kulturkreis hin. Als häufigste Quellen der Entstehung zwischensprachlicher Äquivalente nennt man gewöhnlich die phraseologischen Internationalismen, Entlehnungen bzw. Kalkierungen sowie gleiche Beobachtungen, Erfahrungen, gleiche Lebens- und Denkweise.

Dabei bleiben allerdings bestimmte Fragen unbeantwortet, so z.B. was die Erklärung für die Ähnlichkeit im Sinne von „Bildähnlichkeit” ist; was die Erklärung für die internationale Verbreitung gerade bestimmter Phraseolexeme, und was die Erklärung für phraseologische Entlehnungen sein mag.

Im Rahmen der metaphorischen (und universellen) konzeptuellen Strukturen kann man versuchen, diese Fragen zu beantworten.

Den Ausgangspunkt bildet dabei das Vorhandensein derselben konzeptuellen Strukturen als komplexe mentale Organisationseinheiten und Wissensrepräsentationen - über die Grenzen einzelner Sprachgemeinschaften hinaus. In bezug auf ihre Versprachlichung sind grundsätzlich drei Möglichkeiten denkbar:

3.2.1 Gleiche konzeptuelle Strukturen werden auf die gleiche Art und Weise versprachlicht - im Ergebnis entstehen gleiche Phraseolexeme im Sinne von totalen Äquivalenten. Umgekehrt (vom Ergebnis her) betrachtet: Wenn totale Äquivalente vorliegen, hat man es mit der gleichen Versprachlichung gleicher konzeptueller Strukturen zu tun:

(5) dt. den Faden verlieren - ung. elveszíti a fonalat

(6) dt. sich benehmen wie ein Elefant im Porzellanladen - ung. úgy viselkedik, mint elefánt a porcelánboltban

3.2.2 Gleiche konzeptuelle Strukturen werden auf verschiedener Kongruenzgrundlage auf verschiedene Art und Weise versprachlicht - im Ergebnis entstehen ähnliche Phraseolexeme im Sinne von partieller Äqui- 
valenz. Umgekehrt: Liegen partielle Äquivalente vor, so handelt es sich um die Versprachlichung gleicher konzeptueller Strukturen, nur auf verschiedene Art:

(7) dt. grünes Licht geben - ung. zöld utat ad (wörtlich: „grünen Weg geben")

(8) dt. aus einer Mücke einen Elefanten machen-ung. bolhából elefántot csinál (wörtlich: „aus einem Floh einen Elefanten machen”)

Bei (7) beruht die phraseologische Übertragung auf der Kongruenz zwischen jeweils einem Knoten der Frames „Verkehr” und „Erlaubnis/Möglichkeit", nur im Deutschen wird das Lichtsignal, im Ungarischen das Ergebnis, der Weg/die Straße versprachlicht. Bei (8) wird das antithetische Verhältnis im Deutschen zwischen Mücke und Elefant, im Ungarischen zwischen Floh und Elefant erfaBt.

Die Bildähnlichkeit - umgekehrt die teilweise Verschiedenheit - läßt sich erklären durch Rückgriff auf Roschs Aussage über die allgemeine Struktur semantischer Kategorien bzw. die Einschränkung dieser Aussage, die ich nach Schwarze zitiere:

"Wenn Kategorien von Sprache zu Sprache und von Kultur zu Kultur
verschieden sind, so liegt einer der drei folgenden Fäle vor: entweder
sie unterscheiden sich nur in den "Rändern”, sind aber, hinsichtlich des
Prototyps gleich (dies gilt für Farben und Formen), oder sie beruhen
darauf, daB die Welt, in bezug auf die die Kategorien gebildet werden,
nicht die selbe ist (dies gilt z. B. für natürliche Gattungen und Arte-
fakte), oder es bestand für die Bildung bestimmter Kategorien kein
Anlaß."
(Schwarze 1982, S. 2)

3.2.3 Es gibt eine weitere Kategorie der Ähnlichkeit, wo die Verschiedenheit in der Versprachlichung offenbar außersprachlich, kulturell/historisch bedingt ist:

(9) dt. wie Gott in Frankreich leben- ung. él, mint Marci Hevesen (wörtlich: "leben wie Martin in Heves")

(10) dt. jn. zum Narren halten - ung. lóvá tesz vkit (wörtlich: „jn. zum Pferd machen")

Ob hier auch von verschiedenen konzeptuellen Strukturen auszugehen ist, wage ich nicht zu sagen, wie ich auch für die Faux amis (auch PseudoÄquivalente genannt) nicht einmal eine provisorische Zuordnung bzw. Erklärung in diesem Rahmen finden kann. Es gibt nämlich Phraseolexeme, die in ihrem Bildbereich recht ähnlich, in ihrer phraseologischen Bedeutung dagegen recht verschieden sind: 
(11) dt. jn. auf die Schippe nehmen - ung. lapátra tesz (etwa: 'jn. feuern')

(12) dt. jn. ins Gebet nehmen - ung. imába foglalja a nevét (etwa: 'jm. sehr dankbar sein')

\section{SchluDbemerkungen}

Diese letztgenannten sind allerdings nicht die einzigen Fragen, die noch einer gründlichen Untersuchung und eingehender Diskussion bedürfen. Die Aufzählung könnte fortgesetzt werden z.B. damit, ob man aus dem Fehlen eines Phraseolexems in der einen Sprache, d.h. bei Null-Äquivalenz im Vergleich zu einer bestimmten anderen Sprache, auf das Fehlen einer entsprechenden konzeptuellen Struktur schließen kann. Ich gehe davon aus, $\mathrm{da} B$ eine solche Korrelation nicht angenommen werden sollte, da z.B. zwischen Phraseologie und Wortbildung bzw. überhaupt dem Lexikon der Sprache ein enges Verhältnis der Funktionsteilung besteht. Spätestens an dieser Stelle muß erneut betont werden, daß die vom übrigen Lexikon (völlig) losgelöste Untersuchung der Phraseolexeme nicht vertretbar ist, weil dies dem Verhältnis der beiden Bereiche völlig inadäquat wäre.

In diesem Sinne möchte ich dafür plädieren, daB die Phraseologie als integraler Bestandteil vor allem in die kognitive Semantik mit einbezogen wird - wie es z. B. von Lakoff/Johnson (1980) getan wurde, und wie dafür auch Gréciano bereits vor $10 \mathrm{Jahren}$ plädiert hat (Gréciano 1982).

Aus der Fülle der Fragen konnten nur einige und nur in großen Zügen umrissen werden. Diese gewiß lückenhafte und grobmaschige Darstellung dürfte aber gezeigt haben, da $B$ es durchaus erfolgversprechend ist, die Phraseologie im Rahmen der Kognitiven Linguistik zu untersuchen bzw. sie in die kognitive Semantik zu integrieren. Durch ihre Eigenart als Produkt der sekundären Nomination ließen sich an ihr bestimmte kognitive und semantische Prozesse vielleicht sogar besser als in anderen Bereichen des Lexikons nach vollziehen.

\section{Literatur:}

Baranov, A./Dobrovol'skij, D. (1991): Kognitive Modellierung in der Phraseologie: Zum Problem der Aktuellen Bedeutung. In: Fleischer, W./Große, R./Helbig, G./Lerchner, G. (Hg.): Beiträge zur Erforschung der deutschen Sprache. Bd.10, Leipzig: Bibliographisches Institut. In Kommission bei Niemeyer, Tübingen. S. 112-123.

Bausch, K.-R./Gauger, H.-M. (1971): Zum Werk von Mario Wandruszka. In: Bausch, K.-R./Gauger, H.-M. (Hg.): Interlinguistica. Sprachvergleich und Übersetzung. Festschrift zum 60. Geburtstag von Mario Wandruszka. Tübingen. S. I-XIII. 
Bierwisch, Manfred (1987): Linguistik als kognitive Wissenschaft - Erläuterungen zu einem Forschungsprogramın. In: Zeitschrift für Germanistik 6, S. 645-667.

Burger, H./Buhofer, A./Sialm, A. (Hg.) (1982): Handbuch der Phraseologie. Berlin/New York.

Cernyševa, I.I. (1980): Feste Wortkomplexe des Deutschen in Sprache und Rede. Moskva.

Dobrovol'skij, D. (1988): Phraseologie als Objekt der Universalienlinguistik. Leipzig.

Dobrovol'skij, D. (1992a): Phraseologie und sprachliches Weltbild. Vorarbeiten zum Thesaurus der deutschen Idiomatik. In: Földes, Cs. (Hg.): Deutsche Phraseologie in Sprachsystem und Sprachverwendung. Wien. S. 171-195.

Dobrovol'skij, D. (1992b): Thesaurus deutscher Idiome. Vortragsmanuskript.

Durčo, Peter (1990): Die Interpretation der Phraseologismen aus psycholinguistischer Sicht. In: Folia Linguistica. Acta Societatis Linguisticae Europaeae. Tomus XXIV/1-2, S. 1-22.

Fleischer, Wolfgang (1982): Phraseologie der deutschen Gegenwartssprache. Leipzig.

Fónagy, Iván (1978): Nyelvek a nyelvben [Sprachen in der Sprache]. In: Altalános Nyelvészeti Tanulmányok XII, S. 61-105.

Földes, Csaba (1990): Zur Äquivalenz ungarischer und deutscher Phraseologismen. In: Finnisch-ugrische Forschungen (XLIX), 1-3, S. 169-187.

Földes, Csaba (1991): Farbbezeichnungen als phraseologische Strukturkomponenten im Deutschen, Russischen und Ungarischen. In: Palın, Christine (Hg.): „Europhras 90”. Uppsala. S. 77-89. (Acta Universitatis Upsaliensis. Studia Germanistica Upsaliensia 32).

Gréciano, Gertrud (1982): Zur Semantik der deutschen Idiomatik. In: Zeitschrift für germanistische Linguistik 10, S. 295-316.

Gréciano, Gertrud (1988): Der mentale Charakter des Idiomgebrauchs anhand deutscher und französischer Belege. In: Studien zur Sprachkonfrontation. Materialien der 1. Internationalen Arbeitstagung zum RomanischDeutschen Sprachvergleich. Berlin. S. 34-47. (Linguistische Studien Reihe A 176).

Gréciano, Gertrud (1991): Remotivierung ist textsortenspezifisch. In: Palm, Christine (Hg.): „Europhras 90”. Uppsala. S. 91-100. (Acta Universitatis Upsaliensis. Studia Germanistica Upsaliensia 32).

Herder, Johann Gottfried (1952): Zur Philosophie der Geschichte Bd. 1. Berlin.

Korhonen, Jarmo (1991): Konvergenz und Divergenz in deutscher und finnischer Phraseologie. Zugleich ein Beitrag zur Erlāuterung der Verbreitung und Entlehnung von Idiomen. In: Palm, Christine (Hg.): „Europhras 90”. Uppsala. S. 123-137. (Acta Universitatis Upsaliensis. Studia Germanistica Upsaliensia 32).

Lakoff, G./Johnson, M. (1980): Metaphors We Live by. Chicago/London. 
Möhring, J. (1992): Onomasiologische Verfahren in der Phraseologie. In: Földes, Cs. (Hg.): Deutsche Phraseologie in Sprachsystem und Sprachverwendung. Wien. S. 125-146.

Porzig, Walter (1986): Das Wunder der Sprache. 8. Aufl. Tübingen. (UTB für Wissenschaft: Uni-Taschenbücher 32 ).

Raichštejn, A.D. (1980): Sopostavitel'nyj analiz nemeckoj i russkoj frazeologii. Moskva.

Schwarz, Monika (1992): Einführung in die Kognitive Linguistik. Tübingen. (UTB für Wissenschaft: Uni-Taschenbücher 1636).

Schwarze, Christoph (1982): Stereotyp und lexikalische Bedeutung. Sonderforschungsbereich 99 Linguistik. Universität Konstanz. S. 1-30. (Manuskriptdruck).

Wotjak, Barbara (1992): Verbale Phraseolexeme in System und Text. Tübingen. (Reihe Germanistische Linguistik 125). 